\title{
Current-induced spin torque resonance of a magnetic insulator
}

\author{
Michael Schreier, ${ }^{1,2, *}$ Takahiro Chiba, ${ }^{3}$ Arthur Niedermayr,${ }^{1,2}$ Johannes Lotze, ${ }^{1,2}$ Hans Huebl, ${ }^{1,4}$ Stephan Geprägs, ${ }^{1}$ \\ Saburo Takahashi, ${ }^{3}$ Gerrit E. W. Bauer, ${ }^{3,5,6}$ Rudolf Gross,,${ }^{1,2,4}$ and Sebastian T. B. Goennenwein ${ }^{1,4}$ \\ ${ }^{1}$ Walther-Meißner-Institut, Bayerische Akademie der Wissenschaften, 85748 Garching, Germany \\ ${ }^{2}$ Physik-Department, Technische Universität München, 85748 Garching, Germany \\ ${ }^{3}$ Institute for Materials Research, Tohoku University, Sendai, Miyagi 980-8577, Japan \\ ${ }^{4}$ Nanosystems Initiative Munich, 80799 Munich, Germany \\ ${ }^{5}$ WPI Advanced Institute for Materials Research, Tohoku University, Sendai, Miyagi 980-8577, Japan \\ ${ }^{6}$ Kavli Institute of NanoScience, Delft University of Technology, 2628 CJ Delft, The Netherlands \\ (Received 3 March 2015; revised manuscript received 28 July 2015; published 9 October 2015)
}

\begin{abstract}
We report the observation of current-induced spin torque resonance in yttrium iron garnet/platinum bilayers. An alternating charge current at $\mathrm{GHz}$ frequencies in the platinum gives rise to dc spin pumping and spin Hall magnetoresistance rectification voltages, induced by the Oersted fields of the ac current and the spin Hall effect-mediated spin transfer torque. In ultrathin yttrium iron garnet films, we observe spin transfer torque actuated magnetization dynamics which are significantly larger than those generated by the ac Oersted field. Spin transfer torques thus efficiently couple charge currents and magnetization dynamics also in magnetic insulators, enabling charge current-based interfacing of magnetic insulators with microwave devices.
\end{abstract}

DOI: 10.1103/PhysRevB.92.144411

\section{INTRODUCTION}

Pure spin currents transport angular momentum without an associated charge flow. This makes them attractive for spintronics applications, such as torque-induced magnetization control in nanodevices [1-3], for sensing, data storage, interconnects, and logics. Up to now, however, most spin transfer torque studies focused on metallic ferromagnets [4-8], while magnetic insulators received much less attention [9-11]. However, some magnetic insulators such as yttrium iron garnet (YIG) with extremely low magnetization damping [12] are well suited for the long-range transmission of signals via magnetization dynamics, and may harbor magnon condensates [13] or magnonic crystals [14]. Spin transfer torque and spin pumping [15] provide the communication channel for exploiting insulating magnetic materials in spintronic and electronic devices. Even though a number of phenomena have been attributed to the spin transfer torque acting on magnetic insulators $[9,16]$, the issue is still controversially discussed [17].

Here, we report on the observation of spin torque-induced magnetization dynamics [18] in a magnetic insulator. Applying a microwave-frequency $(\mathrm{GHz})$ charge current to the Pt layer of a YIG/Pt sample, we are able to drive ferromagnetic resonance by the combined action of Oersted fields and spin transfer torque. We observe a large dc voltage when resonant magnetization dynamics are excited in the YIG, which can be accounted for by considering electrically detected dc spin pumping [15] and rectification mediated by the ac spin Hall magnetoresistance (SMR) $[19,20]$. Since the action of Oersted fields and spin transfer torques on spin pumping and SMR rectification are intertwined in a nontrivial fashion, we compare samples with different YIG film thicknesses, which enables us to discern and quantify the magnetization dynamics driven by the two aforementioned excitation processes. Indeed, we can quantitatively model our experimental data using spin diffusion theory and quantum-mechanical interface boundary

*michael.schreier@wmi.badw.de
PACS number(s): 72.25.Mk, 72.25.Ba, 75.70.Tj, 75.40.Gb

conditions [21,22]. Our analysis proves that in very thin YIG films magnetization dynamics indeed are driven by spin transfer torque. In our thinnest YIG film the latter are, in fact, substantially more efficient than the Oersted fields at actuating magnetization dynamics. This opens new perspectives for the efficient integration of ferro-, ferri-, and antiferromagnetic insulators, in the form of, e.g., spin transfer torque magnetic random access memory (STT-MRAM) and spin-wave based interconnects, into electronic devices.

This paper is organized as follows: Since the interplay between the torques on the magnetization and the ensuing $\mathrm{dc}$ voltages is rather subtle, we start with an overview over the relevant theoretical concepts in Sec. II. After introducing the samples and measuring techniques used in Sec. III, we present and analyze the experimental data in Sec. IV. A discussion of the results and our conclusions are given in Secs. V and VI, respectively.

\section{THEORY}

In conventional magnetic resonance studies with coplanar waveguides the Oersted field $B_{\mathrm{ac}}$ generated by a highfrequency current drives the magnetization precession in a nearby ferromagnet. The high-frequency current in our experiment is fed to a thin $\mathrm{Pt}$ film. The resulting high frequency magnetic field can be calculated as $B_{\mathrm{Oe}}=J_{\mathrm{c}} d_{\mathrm{N}} \mu_{0} / 2$, where $J_{\mathrm{c}}$ is the charge current density, $d_{\mathrm{N}}$ is the thickness of the $\mathrm{Pt}$ film, and $\mu_{0}$ is the vacuum permeability. Owing to the spin Hall effect $[23,24]$ a charge current in Pt is accompanied by a transverse spin current. At the ferromagnetic insulator/normal metal interface, this spin current exerts an oscillating spin transfer torque on the magnetization [16] that can drive a magnetization precession. An effective magnetic field can be employed to parametrize the (anti-)damping torque [21] that varies with the angle between spin accumulation and magnetization. Its maximum value is $B_{\mathrm{STT}}=\theta_{\mathrm{SH}} J_{\mathrm{c}} \hbar /\left(2 e M_{\mathrm{S}} d_{\mathrm{F}}\right) \eta$, where $\theta_{\mathrm{SH}}$ is the spin Hall angle, $d_{\mathrm{F}}$ is the thickness of the YIG film and $M_{\mathrm{S}}$ is its saturation magnetization. Furthermore, $\hbar$ is 
the Planck constant, $e>0$ is the elementary charge, and $\eta=$ $2 \lambda \rho \operatorname{Re}\left(G^{\uparrow \downarrow}\right) \tanh \frac{d_{\mathrm{N}}}{2 \lambda} /\left[1+2 \lambda \rho \operatorname{Re}\left(G^{\uparrow \downarrow}\right) \operatorname{coth} \frac{d_{\mathrm{N}}}{\lambda}\right]$ describes the spin absorption efficiency [25] with the spin diffusion length $\lambda$, electrical resistivity $\rho$ in the normal metal, and the spin mixing interface conductance $G^{\uparrow \downarrow}$ (in units of $\Omega^{-1} \mathrm{~m}^{-2}$ ). Conversely, when magnetization dynamics in the ferromagnet are excited, the normal metal provides an additional magnetization damping channel, a process which can be described as injection of a pure spin current from the ferromagnet into the normal metal. The inverse spin Hall effect makes detection of this spin current generated by spin pumping [15] possible in terms of a dc inverse spin Hall voltage $V_{\mathrm{SP}}[26]$. $V_{\mathrm{SP}}$ is a measure of the power absorbed by the magnetic system and therefore yields a purely symmetric resonance line shape [26], irrespective of the origin of the magnetization precession.

The spin transfer torque described above in addition also couples the magnetization orientation of the ferromagnet to the electrical resistivity of the normal metal via the SMR [19]. Owing to SMR the resistivity of the Pt film is modulated by $\Delta \rho_{1}=\rho \theta_{\mathrm{SH}}^{2}\left(\lambda / d_{\mathrm{N}}\right) \eta \tanh \frac{d_{\mathrm{N}}}{2 \lambda}$ as a function of the magnetization orientation in YIG. In magnetic resonance, the high-frequency precession of the magnetization induces a high-frequency oscillation of $\rho$ at the same frequency $f_{\mathrm{MW}}$ as $J_{\mathrm{c}}$, which generates a dc rectification voltage [27] $\left(V_{\mathrm{SMR}}\right)$. In contrast to the symmetric line shape of $V_{\mathrm{SP}}, V_{\mathrm{SMR}}$ also depends on the specifics of the phase relation between microwave current and magnetization precession [28], such that both symmetric and antisymmetric resonance line shapes are possible. For spin torque actuated magnetization dynamics the rectification voltage resonance line shape is purely symmetric [21], while for Oersted field induced dynamics it is, in general, at least partially antisymmetric. Oersted fields, however, are affected by the local sample configuration. This may introduce a (dc magnetic field independent) phase offset $\delta$ between $J_{\mathrm{c}}$ and $\rho$ which can render also the Oersted field induced $V_{\text {SMR }}$ largely symmetric [20,28,29]. Therefore, a simple line-shape analysis of the dc voltage detected in magnetic resonance in terms of symmetric and antisymmetric contributions does not allow us to distinguish the individual contributions from spin pumping and spin Hall magnetoresistance rectification or whether the dynamics stem from Oersted fields or the spin transfer torque. Thus, in order to assess the importance of the spin torque actuated dynamics, a more careful and elaborate quantitative analysis has to be performed. The corresponding expressions for $V_{\mathrm{SP}}$ and $V_{\mathrm{SMR}}$ are discussed in the remainder of this section. As a general guideline, however, the spin transfer torque manifests itself as an increasingly symmetric resonance line shape of $V_{\mathrm{dc}}=V_{\mathrm{SP}}+V_{\mathrm{SMR}}$ for decreasing thickness of the ferromagnet.

The mathematical modeling of the ac-dc conversion mechanism is discussed in detail in Refs. [21,22]. Converting notation to SI units, the dc spin pumping and rectification voltage read

$$
\begin{aligned}
V_{\mathrm{SP}} & =S_{\mathrm{SP}} \frac{\Delta B^{2}}{\left(B_{\mathrm{ex}}-B_{\mathrm{res}}\right)^{2}+\Delta B^{2}} \cos \varphi \sin 2 \varphi, \\
V_{\mathrm{SMR}}= & {\left[S_{\mathrm{SMR}} \frac{\Delta B^{2}}{\left(B-B_{\mathrm{res}}\right)^{2}+\Delta B^{2}}\right.} \\
& \left.+A_{\mathrm{SMR}} \frac{\left(B_{\mathrm{ex}}-B_{\mathrm{res}}\right) \Delta B}{\left(B_{\mathrm{ex}}-B_{\mathrm{res}}\right)^{2}+\Delta B^{2}}\right] \cos \varphi \sin 2 \varphi,
\end{aligned}
$$

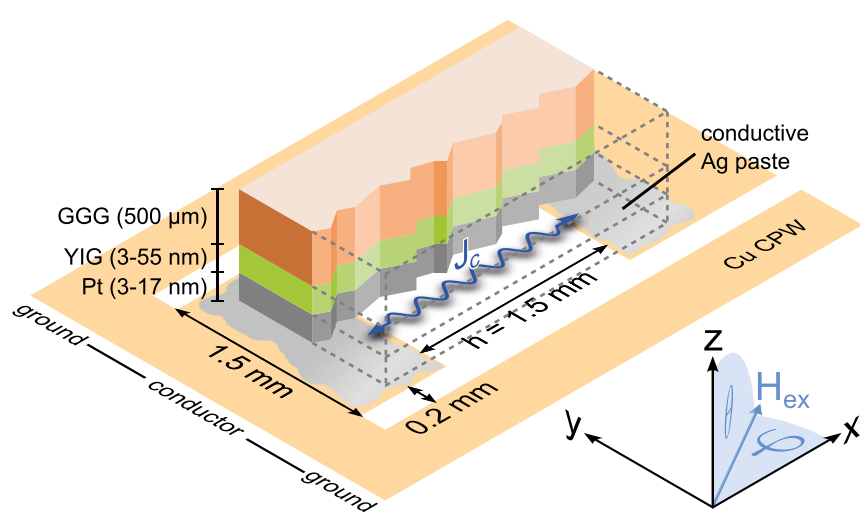

FIG. 1. (Color online) Samples are placed across a gap in the center conductor of a coplanar waveguide and contacted with conductive Ag paste.

where

$$
\begin{aligned}
S_{\mathrm{SP}}= & \frac{h \rho J_{\mathrm{r}}^{\mathrm{P}} C}{4 \Delta B^{2}}\left[C_{+} B_{\mathrm{Oe}}^{2}+C_{-} B_{\mathrm{STT}}^{2}\right. \\
& \left.+C\left(\left(2+\alpha^{2}\right) \sin \delta-\frac{\alpha}{\tilde{\omega}_{\mathrm{a}}} \cos \delta\right) B_{\mathrm{Oe}} B_{\mathrm{STT}}\right], \\
S_{\mathrm{SMR}}= & -\frac{h \Delta \rho_{1} J_{\mathrm{c}}}{4 \Delta B}\left[C B_{\mathrm{STT}}+\left(C_{+} \sin \delta+\alpha C \cos \delta\right) B_{\mathrm{Oe}}\right], \\
A_{\mathrm{SMR}}= & -\frac{h \Delta \rho_{1} J_{\mathrm{c}}}{4 \Delta B}\left(C_{+} \cos \delta-\alpha C \sin \delta\right) B_{\mathrm{Oe}} .
\end{aligned}
$$

Before going into details below we point out that the dc spin pumping voltage $V_{\mathrm{SP}}$ takes the form of a symmetric Lorentzian with magnitude $S_{\mathrm{SP}}$ governed by the Oersted field $B_{\mathrm{Oe}}$ as well as the effective magnetic field parametrizing the (anti-)damping torque $B_{\mathrm{STT}}$. On the other hand, the dc rectification voltage $V_{\mathrm{SMR}}$ is composed of a symmetric and an antisymmetric Lorentzian with magnitude $S_{\mathrm{SMR}}$ and $A_{\mathrm{SMR}}$, respectively; the former depends on both $B_{\mathrm{Oe}}$ and $B_{\mathrm{STT}}$ while the latter only depends on $B_{\mathrm{Oe}}$.

The remaining parameters in Eqs. (1) and (2) are as follows: $h$ is the length of the sample, $\varphi$ is the angle between the current and the applied in-plane magnetic field (see Fig. 1) with modulus $B_{\mathrm{ex}}=\mu_{0} H_{\mathrm{ex}}, \delta$ is the constant phase offset between microwave current and magnetization precession [20,28,29] as discussed above, and the magnetic field $\Delta B=\alpha \omega_{\mathrm{a}} / \gamma$ is the half width of the resonance governed by the Gilbert damping constant $\alpha$ and the excitation angular frequency $\omega_{\mathrm{a}}=2 \pi f_{\mathrm{MW}} . C=\tilde{\omega}_{\mathrm{a}} / \sqrt{1+\tilde{\omega}_{\mathrm{a}}^{2}}$ and $C_{ \pm}=1 \pm 1 / \sqrt{1+\tilde{\omega}_{\mathrm{a}}^{2}}$ where $\tilde{\omega}_{\mathrm{a}}=2 \omega_{\mathrm{a}} /\left(M_{\mathrm{s}} \gamma \mu_{0}\right)$ with the gyromagnetic ratio $\gamma$. Furthermore, $J_{\mathrm{r}}^{\mathrm{P}}=\hbar \omega_{\mathrm{a}} /\left(2 e d_{\mathrm{N}} \rho\right) \theta_{\mathrm{SH}} \eta$ and $B_{\text {res }}=-M_{\mathrm{s}} \mu_{0} / 2+$ $\sqrt{\left(M_{\mathrm{s}} \mu_{0} / 2\right)^{2}+\left(\omega_{\mathrm{a}} / \gamma\right)^{2}}$. Finally, the total damping is given by $\alpha=\alpha_{0}+\gamma \hbar^{2} /\left(2 e^{2} M_{\mathrm{s}} d_{\mathrm{F}}\right) \operatorname{Re}\left[G^{\uparrow \downarrow} /\left(1+2 \rho \lambda G^{\uparrow \downarrow} \operatorname{coth} \frac{d_{\mathrm{N}}}{\lambda}\right)\right]$, where the parameter $\alpha_{0}$ combines the intrinsic Gilbert damping of the YIG film with its inhomogeneous broadening.

\section{SAMPLE PREPARATION AND EXPERIMENTAL METHODS}

YIG films were grown in oxygen atmosphere at a pressure of $25 \mu$ bar on (111) oriented 500- $\mu \mathrm{m}$-thick gadolinium gallium garnet (GGG) substrates by laser molecular beam epitaxy. 
Subsequently the Pt layer was deposited in situ, without breaking the vacuum, on the YIG thin film by electron-beam evaporation [30]. The resistivity of the Pt films varies within the margin expected from earlier measurements [30]. In very thin films we routinely observe a variation of the resistivity by a factor of 2 due to surface termination, ambient pressure, and other growth related variables. The YIG/Pt samples are integrated into a coplanar waveguide (CPW) structure with a characteristic impedance of $50 \Omega$ and placed onto a $1.5-\mathrm{mm}-$ wide gap in the center conductor [31]. The sample dimensions are designed to impedance match the microwave circuitry, leading to an effective sample area of about $1.5 \times 1.5 \mathrm{~mm}$. The CPW with the integrated sample is placed between the pole shoes of a rotatable electromagnet. In the experiments, we use an intensity modulated ( $\left.f_{\text {mod }} \cong 10 \mathrm{kHz}\right)$ microwave source $\left(f_{\mathrm{MW}}=7 \mathrm{GHz}\right)$ to feed the samples with an ac charge current. The ensuing dc voltages are detected by a lock-in amplifier. All experiments were performed under ambient conditions.

\section{EXPERIMENTAL RESULTS}

\section{A. In-plane measurements and simulations}

We performed the ferromagnetic resonance studies for three samples with strategically chosen film thicknesses [YIG(55 nm)/Pt(17 nm), YIG(55 nm)/Pt(4 nm), and YIG $(4 \mathrm{~nm}) / \mathrm{Pt}(3 \mathrm{~nm})]$ which allows us to disentangle Oersted and spin transfer torque contributions to $V_{\mathrm{dc}}$.

As shown in Fig. 2(a) we observe a mostly symmetric negative voltage peak at the resonance magnetic field $\mu_{0} H_{\mathrm{ex}} \approx 0.18 \mathrm{~T}$ for the $\mathrm{YIG}(55 \mathrm{~nm}) / \operatorname{Pt}(17 \mathrm{~nm})$ sample. To evaluate the importance of the spin transfer torque we simulate the experimentally observed voltage using the model described in Sec. II [Eqs. (1) and (2)]. We note that the large and partly correlated parameter space of Eqs. (1) and (2) adversely affects the accuracy of the numerical values given in the following. Nevertheless, any plausible parameter set will qualitatively yield very similar and thus robust results regarding the contributions of Oersted field and spin transfer torque. Moreover, most parameters are constrained (by literature or other measurements), leaving only charge current density $J_{\mathrm{c}}$ and intrinsic damping parameter $\alpha_{0}$ as free parameters in the model calculations. These, however, can also be estimated well from the applied microwave source power, impedance matching characteristics, and the damping parameters extracted from spin pumping experiments on our YIG/Pt samples [32]. The spin transport parameters are spin diffusion length $\lambda=1.5 \mathrm{~nm}$, spin Hall angle $\theta_{\mathrm{SH}}=0.11$, and spin mixing conductance $\operatorname{Re}\left(G^{\uparrow \downarrow}\right)=4 \times 10^{14} \Omega^{-1} \mathrm{~m}^{-2}$, which have proven to be robust to variations of other sample parameters (Refs. [30,32]). The phase $\delta=-63^{\circ}$ is inferred from additional measurements with magnetic fields oriented at a slight angle to the film normal (see Sec. IV B). The saturation magnetization $M_{\mathrm{s}}=118 \mathrm{kA} / \mathrm{m}$ is determined from the magnetic resonance field, and $\rho=445 \times 10^{-9} \Omega \mathrm{m}$ from dc resistance measurements. For $J_{\mathrm{c}}=0.53 \times 10^{9} \mathrm{~A} / \mathrm{m}^{2}$ and $\alpha_{0}=0.01$, we obtain good quantitative agreement between model and experiment [Fig. 2(a)]. The large effective intrinsic damping $\alpha_{0}$ in our samples can be understood in terms of efficient two-magnon scattering induced by roughness in thin
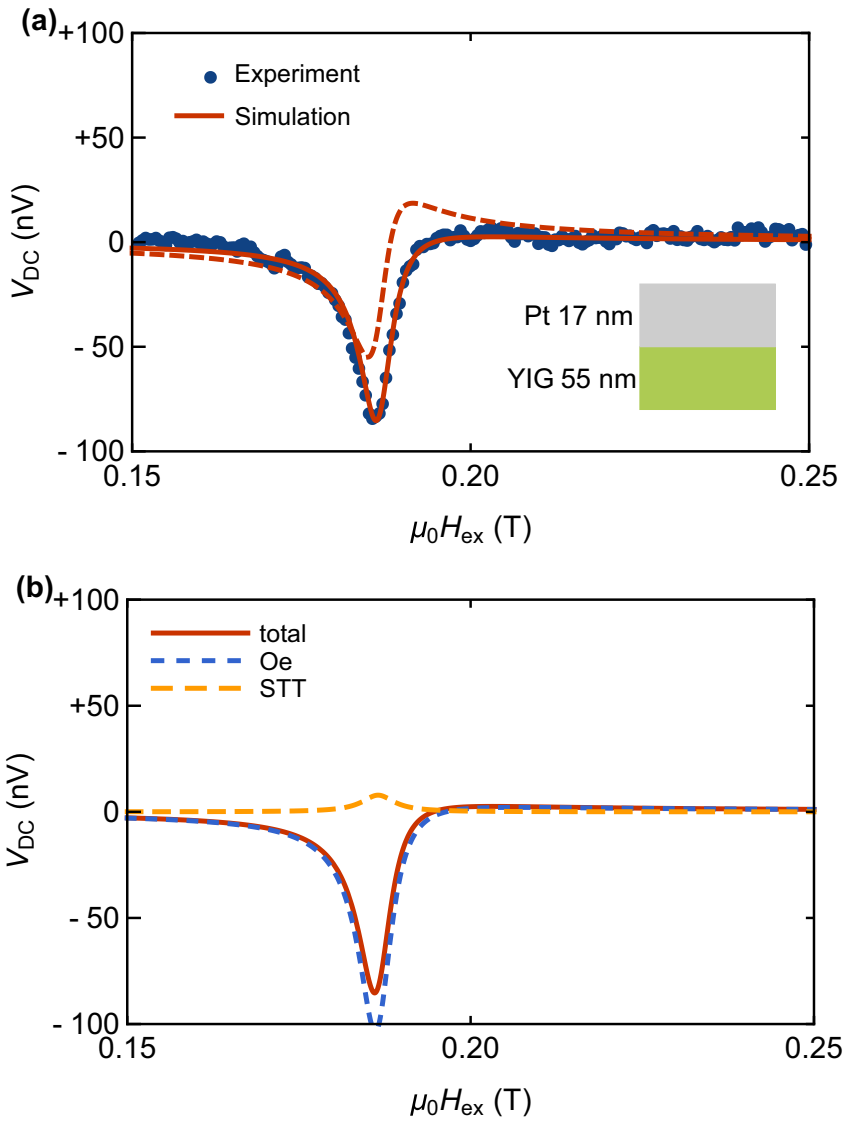

FIG. 2. (Color online) (a) Measured dc voltage $V_{\mathrm{dc}}$ of YIG(55 nm)/Pt(17 nm) under an ac current bias (full symbols). The solid/dashed line is calculated from a simulation based on Eqs. (1) and (2) using $\delta=-63^{\circ} / \delta=0$, respectively. The angle between the (in-plane) external magnetic field and the microwave current is $\varphi \cong-35^{\circ}\left(\theta=90^{\circ}\right.$, Fig. 1). (b) Contributions to the excitation by Oersted field and spin transfer torque to $V_{\mathrm{dc}}$, according to the simulation. The Oersted fields dominate the magnetization actuation.

ferromagnetic films $[33,34]$, especially when magnetized in the film plane [35].

After finding good agreement of the simulated and the measured $V_{\mathrm{dc}}$ we may analyze the contributions due to the Oersted field and spin transfer torque in Fig. 2(b) in the simulation. We find that the excitation in this sample is dominated by the Oersted field as expected for the comparably large YIG layer thickness. The largely symmetric shape of the resonance peak is due to a significant spin pumping contribution, with SMR being small $\left(\Delta \rho / \rho \approx 2 \times 10^{-4}\right)[19,30]$ for thick Pt films.

In the $\mathrm{YIG}(55 \mathrm{~nm} /) \mathrm{Pt}(4 \mathrm{~nm})$ sample with its smaller $\mathrm{Pt}$ thickness, we observe a distinct asymmetric $V_{\mathrm{dc}}$ resonance line shape [Fig. 3(a)]. This particular sample shows a somewhat higher resonance field and absolute voltage level due to a reduced saturation magnetization and better impedance matching, respectively. Again, the experimental data are well reproduced by the simulation using $M_{\mathrm{s}}=89 \mathrm{kA} / \mathrm{m}, J_{\mathrm{c}}=4 \times$ $10^{9} \mathrm{~A} / \mathrm{m}^{2}, \rho=317 \times 10^{-9} \Omega \mathrm{m}, \delta=-55^{\circ}$, and $\alpha_{0}=0.015$. The SMR is known to be maximal for a Pt thickness of roughly twice the spin diffusion length [30] $d_{\mathrm{N}} \approx 2 \lambda=3 \mathrm{~nm}$. The antisymmetric shape here then is due to a significantly increased SMR rectification as compared to the $\mathrm{YIG}(55 \mathrm{~nm}) / \mathrm{Pt}(17 \mathrm{~nm})$ 

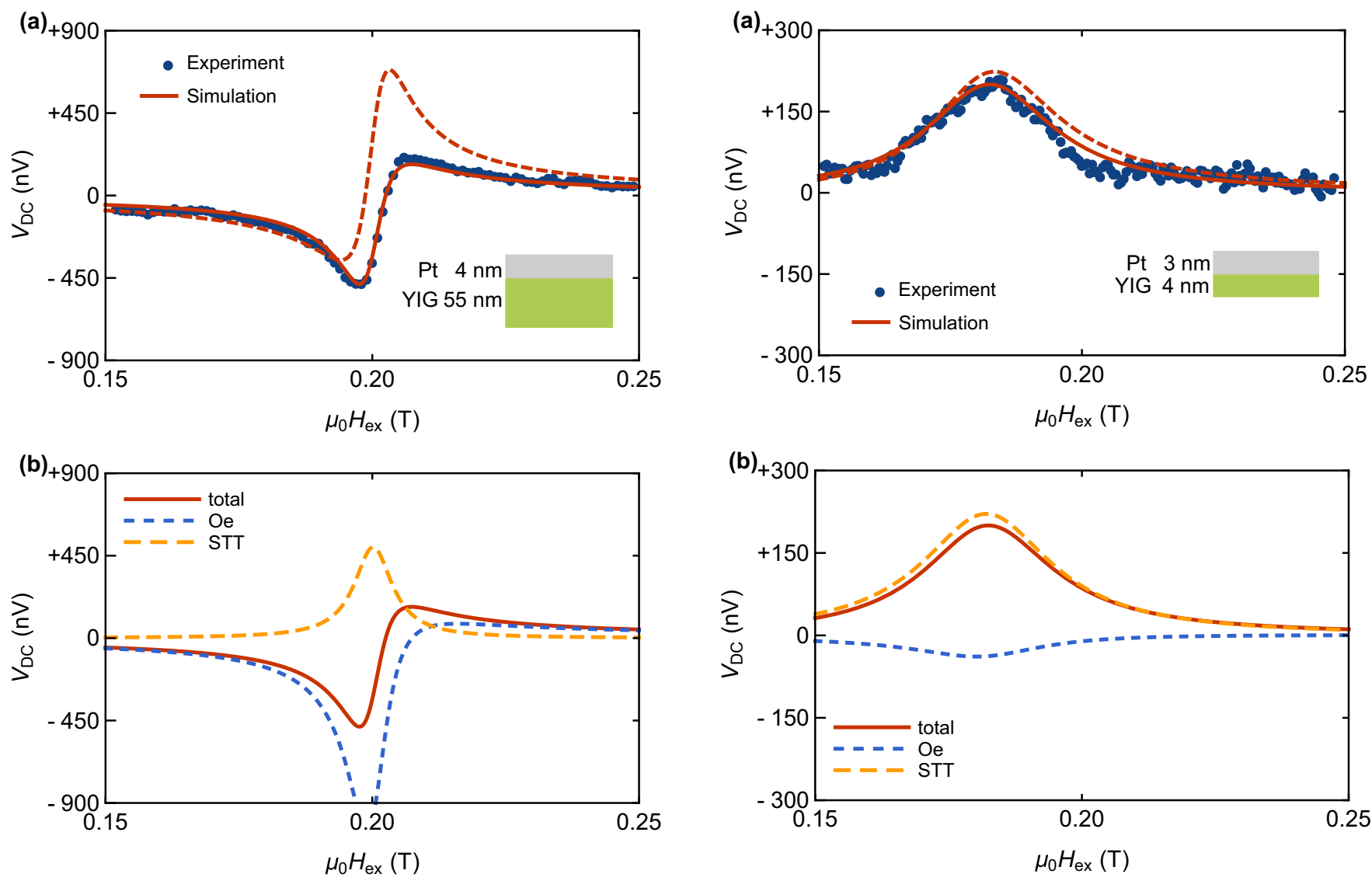

FIG. 3. (Color online) (a) Measured dc voltage $V_{\mathrm{dc}}$ of YIG(55 nm)/Pt(4 nm) under an ac current bias (full symbols). The solid/dashed line is calculated from a simulation based on Eqs. (1) and (2) using $\delta=-55^{\circ} / \delta=0$, respectively. The angle between the (inplane) external magnetic field and the microwave current is $\varphi \cong-35^{\circ}$ $\left(\theta=90^{\circ}\right.$, Fig. 1). (b) Contributions to the excitation by Oersted field and spin transfer torque to $V_{\mathrm{dc}}$, according to the simulation. While the smaller $d_{\mathrm{N}}$ benefits the effective field describing the (anti-)damping torque the magnetization dynamics are still dominantly induced by the Oersted field.

sample. The smaller $d_{\mathrm{N}}$ also enhances the effective field describing the (anti-)damping torque. However, since the YIG layer is still comparably thick, the Oersted field contribution still dominates the excitation [Fig. 3(b)].

The YIG(4 nm)/Pt(3 nm) sample behaves markedly different. Here, we observe a broad positive voltage peak which indicates STT excitation [Fig. 4(a)]. With decreasing YIG film thickness, the effect of surface roughness on the magnetization damping is increased. This is taken care of in our simulations with $M_{\mathrm{s}}=128 \mathrm{kA} / \mathrm{m}, J_{\mathrm{c}}=1.1 \times 10^{9} \mathrm{~A} / \mathrm{m}^{2}, \rho=$ $481 \times 10^{-9} \Omega \mathrm{m}, \delta=-78^{\circ}$, and $\alpha_{0}=0.04$. The symmetric line shape in Fig. 4(b) is caused by the dominant spin transfer torque component.

\section{B. Out-of-plane measurements and extraction of the phase offset $\delta$}

As discussed in Sec. IV A, the measured dc voltages, especially their dependence on the thickness of the YIG and Pt layer, are well reproduced by theory. As seen in Figs. 2-4,

FIG. 4. (Color online) (a) Measured dc voltage $V_{\mathrm{dc}}$ of YIG(4 nm)/Pt(3 nm) under an ac current bias (full symbols). The solid/dashed line is calculated from a simulation based on Eqs. (1) and (2) using $\delta=-78^{\circ} / \delta=0$, respectively. The angle between the (in-plane) external magnetic field and the microwave current is $\varphi \cong-35^{\circ}\left(\theta=90^{\circ}\right.$, Fig. 1). (b) Contributions to the excitation by Oersted field and spin transfer torque to $V_{\mathrm{dc}}$, according to the simulation. The spin transfer torque dominates the excitation of the magnetization dynamics due to the very thin YIG layer.

the best match between theory and experiment is achieved, however, only by allowing for $\delta \neq 0$ in Eqs. (1) and (2). Although good agreement is obtained for $\delta \neq 0$, one has to keep in mind that the Oersted field-induced SMR rectification voltage [Eq. (2)] then also contributes to the symmetric line shape. Therefore, the phase offset $\delta$ must be determined in order to conclusively establish the role of the spin transfer torque versus the Oersted field. While both $V_{\mathrm{SP}}$ and $V_{\mathrm{SMR}}$ vanish for magnetic fields in the plane spanned by the film normal and the charge current direction [28], i.e., $\varphi=0^{\circ}$, for any other value of $\varphi$ their magnitude is differently affected by the polar (out-of-plane) angle $\theta$ between the film normal and the magnetization direction [22]. More specifically, the ratio $R$ of the symmetric $(S)$ to antisymmetric $(A)$ contributions to the line shape [20] changes characteristically as a function of $\theta$ for a given $\delta$. A pronounced change in $R$ is observed for $\theta \rightarrow 0^{\circ}$, i.e., for magnetization nearly perpendicular to the film plane. Since the dc voltage vanishes for $\theta=0^{\circ}$, however, it is not possible to take data with the magnetic field exactly perpendicular to the film stack. For all samples we thus carried out additional experiments (Fig. 5) with the magnetic 


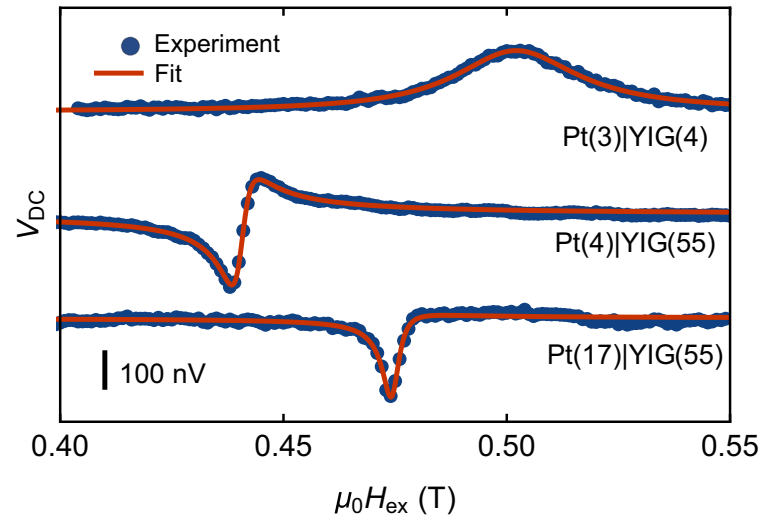

FIG. 5. (Color online) Measured and fitted dc voltages for external fields applied at a slight angle $\theta \approx 5^{\circ}$ relative to the film normal on the $\mathrm{YIG}(55 \mathrm{~nm}) / \mathrm{Pt}(17 \mathrm{~nm}), \mathrm{YIG}(55 \mathrm{~nm}) / \mathrm{Pt}(4 \mathrm{~nm})$, and YIG(4 nm)/Pt(3 nm) sample, respectively.

field applied at a small angle to the film normal $\left(\theta \approx 5^{\circ}\right.$, $\varphi=90^{\circ}$, Fig. 1). A direct computation of the experimental out-of-plane resonance spectra is unfortunately not possible as magnetocrystalline anisotropy other than shape anisotropy is not accounted for in Refs. [21,22] but affects the resonance field in the out-of-plane measurements. The measured value may further be affected by the different sample position in the magnet for the out-of-plane configuration. The line shape itself, however, is hardly affected for typical crystalline anisotropy strengths in our thin films. The phase offset $\delta$ can then be determined by extracting $R$ for the in-plane and out-of-plane measurements and adjusting $\delta$ such that both the in-plane and out-of-plane $R$ value are reproduced. This procedure is essentially the same as introducing an artificial resonance field offset in the simulation to counteract the effect of the crystalline anisotropy which amounts up to a few $\mathrm{mT}$. $R=S / A$ is obtained by fitting the experimental data assuming a generalized Lorentzian, i.e.,

$$
V_{\mathrm{dc}}=S \frac{\Delta B^{2}}{\left(B_{\mathrm{ex}}-B_{\mathrm{res}}\right)^{2}+\Delta B^{2}}+A \frac{\Delta B\left(B_{\mathrm{ex}}-B_{\mathrm{res}}\right)}{\left(B_{\mathrm{ex}}-B_{\mathrm{res}}\right)^{2}+\Delta B^{2}},
$$

where $\Delta B$ is the linewidth, $B_{\mathrm{res}}$ is the resonance field, $B_{\mathrm{ex}}$ is the external field, and $S$ and $A$ are the amplitudes of the symmetric and antisymmetric contributions to the line shape. For the simulation we disregarded all terms associated with the imaginary part of the spin mixing conductance and assumed that the static magnetization is oriented along the external field direction. Since the out-of-plane magnetic resonance fields are of the order of $450 \mathrm{mT}$ (cf. $\mu_{0} M_{\mathrm{s}} \lesssim 160 \mathrm{mT}$ ), $\operatorname{Re}\left(G^{\uparrow \downarrow}\right) \gg$ $\operatorname{Im}\left(G^{\uparrow \downarrow}\right)$ in our YIG/Pt samples [30], and the Oersted as well as the effective field describing the (anti-)damping torque both lie in the film plane when $\theta \rightarrow 0^{\circ}$, these assumptions are not expected to compromise our results.

The experimental $R$ values are found as $R_{\mathrm{ip}}=-3.2$, $R_{\text {oop }}=-2.5$ for the YIG $(55 \mathrm{~nm}) / \operatorname{Pt}(17 \mathrm{~nm})$ sample; $R_{\text {ip }}=$ $-0.43, R_{\text {oop }}=-0.35$ for the $\mathrm{YIG}(55 \mathrm{~nm}) / \operatorname{Pt}(4 \mathrm{~nm})$ sample; and $R_{\text {ip }}=19, R_{\text {oop }}=25$ for the YIG $(4 \mathrm{~nm}) / \operatorname{Pt}(3 \mathrm{~nm})$ sample. The subscripts ip and oop indicate magnetic field in the film plane and (approximately) out of plane, respectively.
A comparison with the simulation based on Ref. [22] then yields phase offsets of $\delta=-63^{\circ}$ [YIG(55 nm)/Pt(17 nm)], $\delta=-55^{\circ} \quad[\mathrm{YIG}(55 \mathrm{~nm}) / \operatorname{Pt}(4 \mathrm{~nm})], \quad$ and $\quad \delta=-78^{\circ}$ [YIG(4 $\mathrm{nm}) / \operatorname{Pt}(3 \mathrm{~nm})]$, respectively. The $R$ value of the $\mathrm{YIG}(4 \mathrm{~nm}) / \mathrm{Pt}(3 \mathrm{~nm})$ sample is most uncertain, causing a phase offset error of about $\pm 3^{\circ}$.

\section{SUMMARY AND DISCUSSION}

The pronounced dependence of the $V_{\mathrm{dc}}$ resonance spectra on the thicknesses of the yttrium iron garnet and platinum layers $\left(d_{\mathrm{F}}\right.$ and $\left.d_{\mathrm{N}}\right)$, observed in experiment and accurately reproduced by the model, provides clear evidence for spin transfer torque driven magnetization dynamics in thin yttrium iron garnet films. From a different perspective, our results also show that ac spin pumping [36-38] in magnetic insulators is reciprocal, as predicted by Onsager symmetry in the linear-response regime. Spin transfer torque therefore provides an efficient link between pure magnonic and conventional electronic circuits. While Oersted fields drive bulk magnetization dynamics, the spin transfer torque is linked to the interface and is thus very effective for thin film structures. Magnetic insulators in particular can be engineered for low saturation magnetization [39], further increasing the effectiveness of spin transfer torque mediated magnetization actuation. Recent results $[10,11]$ also indicate that the spin transfer torque may be able to couple to the Néel order parameter in antiferromagnetic insulators which could allow for very high-frequency dynamics. The origin of the phase offset $\delta$ is not known. It could originate from our particular measurement geometry, electromagnetic wave propagation through the magnetic medium, a frequency dependent spin Hall angle as recently proposed by Weiler et al. [38], or a significant current-induced effective field torque $[40,41]$.

\section{CONCLUSION}

In conclusion, we studied resonant magnetization precession induced by an ac charge current in yttrium iron garnet/platinum bilayers. The induced ferromagnetic resonance was detected via a combination of spin pumping-induced inverse spin Hall effect and spin Hall magnetoresistance rectification voltages. Using samples with different yttrium iron garnet and platinum layer thicknesses we could disentangle and quantify the spin torque contribution to the magnetization dynamics. Our modeling of the dc voltage observed at resonance strongly suggests that spin transfer torque efficiently actuates the magnetization in thin yttrium iron garnet films.

\section{ACKNOWLEDGMENTS}

We thank Sibylle Meyer, Michaela Lammel, and Stephan Altmannshofer for the fabrication of YIG/Pt samples. Financial support from the Deutsche Forschungsgemeinschaft via SPP Grant No. 1538 "Spin Caloric Transport," Project No. GO 944/4-2, Project No. BA 2954/1-2, FOM (Stichting voor Fundamenteel Onderzoek der Materie), the ICC-IMR, EU-FET InSpin Grant No. 612759, and Grants-in-Aid for Scientific Research (KAKENHI) No. 22540346, No. 25247056, No. 25220910, and No. 268063 is gratefully acknowledged. 
[1] I. M. Miron, K. Garello, G. Gaudin, P.-J. Zermatten, M. V. Costache, S. Auffret, S. Bandiera, B. Rodmacq, A. Schuhl, and P. Gambardella, Nature (London) 476, 189 (2011).

[2] C.-F. Pai, L. Liu, Y. Li, H. Tseng, D. Ralph, and R. Buhrman, Appl. Phys. Lett. 101, 122404 (2012).

[3] L. Liu, C.-F. Pai, Y. Li, H. W. Tseng, D. C. Ralph, and R. A. Buhrman, Science 336, 555 (2012).

[4] V. E. Demidov, S. Urazhdin, H. Ulrichs, V. Tiberkevich, A. Slavin, D. Baither, G. Schmitz, and S. O. Demokritov, Nat. Mater. 11, 1028 (2012).

[5] P. P. J. Haazen, E. Murè, J. H. Franken, R. Lavrijsen, H. J. M. Swagten, and B. Koopmans, Nat. Mater. 12, 299 (2013).

[6] R. H. Liu, W. L. Lim, and S. Urazhdin, Phys. Rev. Lett. 110, 147601 (2013).

[7] J. Ryu, K.-J. Lee, and H.-W. Lee, Appl. Phys. Lett. 102, 172404 (2013).

[8] S. Kasai, K. Kondou, H. Sukegawa, S. Mitani, K. Tsukagoshi, and Y. Otani, Appl. Phys. Lett. 104, 092408 (2014).

[9] Y. Kajiwara, K. Harii, S. Takahashi, J. Ohe, K. Uchida, M. Mizuguchi, H. Umezawa, H. Kawai, K. Ando, K. Takanashi, S. Maekawa, and E. Saitoh, Nature (London) 464, 262 (2010).

[10] A. Hamadeh, O. d'Allivy Kelly, C. Hahn, H. Meley, R. Bernard, A. H. Molpeceres, V. V. Naletov, M. Viret, A. Anane, V. Cros, S. O. Demokritov, J. L. Prieto, M. Muñoz, G. de Loubens, and O. Klein, Phys. Rev. Lett. 113, 197203 (2014).

[11] T. Moriyama, S. Takei, M. Nagata, Y. Yoshimura, N. Matsuzaki, T. Terashima, Y. Tserkovnyak, and T. Ono, Appl. Phys. Lett. 106, 162406 (2015).

[12] V. Cherepanov, I. Kolokolov, and V. L'vov, Phys. Rep. 229, 81 (1993).

[13] S. O. Demokritov, V. E. Demidov, O. Dzyapko, G. A. Melkov, A. A. Serga, B. Hillebrands, and A. N. Slavin, Nature (London) 443, 430 (2006).

[14] Y. Gulyaev, S. Nikitov, L. Zhivotovskii, A. Klimov, P. Tailhades, L. Presmanes, C. Bonningue, C. Tsai, S. Vysotskii, and Y. Filimonov, JETP Lett. 77, 567 (2003).

[15] Y. Tserkovnyak, A. Brataas, and G. E. W. Bauer, Phys. Rev. Lett. 88, 117601 (2002).

[16] Y.-T. Chen, S. Takahashi, H. Nakayama, M. Althammer, S. T. B. Goennenwein, E. Saitoh, and G. E. W. Bauer, Phys. Rev. B 87, 144411 (2013).

[17] B. F. Miao, S. Y. Huang, D. Qu, and C. L. Chien, Phys. Rev. Lett. 112, 236601 (2014).

[18] L. Liu, T. Moriyama, D. C. Ralph, and R. A. Buhrman, Phys. Rev. Lett. 106, 036601 (2011).

[19] H. Nakayama, M. Althammer, Y.-T. Chen, K. Uchida, Y. Kajiwara, D. Kikuchi, T. Ohtani, S. Geprägs, M. Opel, S. Takahashi, R. Gross, G. E. W. Bauer, S. T. B. Goennenwein, and E. Saitoh, Phys. Rev. Lett. 110, 206601 (2013).

[20] R. Iguchi, K. Sato, D. Hirobe, S. Daimon, and E. Saitoh, Appl. Phys. Expr. 7, 013003 (2014).
[21] T. Chiba, G. E. W. Bauer, and S. Takahashi, Phys. Rev. Applied 2, 034003 (2014).

[22] T. Chiba, M. Schreier, G. E. W. Bauer, and S. Takahashi, J. Appl. Phys. 117, 17C715 (2015).

[23] M. I. D'yakonov and V. I. Perel', JETP Lett. 13, 467 (1971).

[24] J. E. Hirsch, Phys. Rev. Lett. 83, 1834 (1999).

[25] H. J. Jiao and G. E. W. Bauer, Phys. Rev. Lett. 110, 217602 (2013).

[26] O. Mosendz, J. E. Pearson, F. Y. Fradin, G. E. W. Bauer, S. D. Bader, and A. Hoffmann, Phys. Rev. Lett. 104, 046601 (2010).

[27] H. J. Juretschke, J. Appl. Phys. 31, 1401 (1960).

[28] L. Bai, P. Hyde, Y. S. Gui, C.-M. Hu, V. Vlaminck, J. E. Pearson, S. D. Bader, and A. Hoffmann, Phys. Rev. Lett. 111, 217602 (2013).

[29] A. Azevedo, L. H. Vilela-Leão, R. L. Rodríguez-Suárez, A. F. Lacerda Santos, and S. M. Rezende, Phys. Rev. B 83, 144402 (2011).

[30] M. Althammer, S. Meyer, H. Nakayama, M. Schreier, S. Altmannshofer, M. Weiler, H. Huebl, S. Geprägs, M. Opel, R. Gross, D. Meier, C. Klewe, T. Kuschel, J.-M. Schmalhorst, G. Reiss, L. Shen, A. Gupta, Y.-T. Chen, G. E. W. Bauer, E. Saitoh, and S. T. B. Goennenwein, Phys. Rev. B 87, 224401 (2013).

[31] J. Lotze, H. Huebl, R. Gross, and S. T. B. Goennenwein, Phys. Rev. B 90, 174419 (2014).

[32] M. Weiler, M. Althammer, M. Schreier, J. Lotze, M. Pernpeintner, S. Meyer, H. Huebl, R. Gross, A. Kamra, J. Xiao, Y.-T. Chen, H. J. Jiao, G. E. W. Bauer, and S. T. B. Goennenwein, Phys. Rev. Lett. 111, 176601 (2013).

[33] A. Azevedo, A. B. Oliveira, F. M. de Aguiar, and S. M. Rezende, Phys. Rev. B 62, 5331 (2000).

[34] K. Zakeri, J. Lindner, I. Barsukov, R. Meckenstock, M. Farle, U. von Hörsten, H. Wende, W. Keune, J. Rocker, S. S. Kalarickal, K. Lenz, W. Kuch, K. Baberschke, and Z. Frait, Phys. Rev. B 76, 104416 (2007)

[35] R. D. McMichael, M. D. Stiles, P. J. Chen, and W. F. Egelhoff, J. Appl. Phys. 83, 7037 (1998).

[36] C. Hahn, G. de Loubens, M. Viret, O. Klein, V. V. Naletov, and J. Ben Youssef, Phys. Rev. Lett. 111, 217204 (2013).

[37] D. Wei, M. Obstbaum, M. Ribow, C. H. Back, and G. Woltersdorf, Nat. Commun. 5, 3768 (2014).

[38] M. Weiler, J. M. Shaw, H. T. Nembach, and T. J. Silva, Phys. Rev. Lett. 113, 157204 (2014).

[39] S. Geller, J. P. Remeika, R. C. Sherwood, H. J. Williams, and G. P. Espinosa, Phys. Rev. 137, A1034 (1965).

[40] J. Sklenar, W. Zhang, M. B. Jungfleisch, W. Jiang, H. Chang, J. E. Pearson, M. Wu, J. B. Ketterson, and A. Hoffmann, arXiv:1505.07791.

[41] T. Chiba, G. E. Bauer, and S. Takahashi, J. Magn. Magn. Mater., doi: 10.1016/j.jmmm.2015.07.058 (2015). 